\title{
Article
}

\section{Mathematical modeling of rogue waves, a review of conventional and emerging mathematical methods and solutions.}

\author{
Sergio Manzetti ${ }^{t, \ddagger}$ (1)* \\ 1 Uppsala University, BMC, Dept Mol. Cell Biol, Box 596, SE-75124 Uppsala. Sweden; email: \\ sergio.manzetti@icm.uu.se \\ 2 Fjordforsk A/S, Midtun, 6894 Vangsnes. Norway; email: sergio.manzetti@fjordforsk.no \\ * Correspondence: sergio.manzetti@fjordforsk.no; Tel.: +47-4017-6707 \\ † Current address: Fjordforsk A/S, Midtun, 6894 Vangsnes
}

\begin{abstract}
Anomalous waves and rogue events are closely associated with irregularities and unexpected events occurring at various levels of physics, such as in optics, in oceans and in the atmosphere. Mathematical modeling of rogue waves is a highly actual field of research, which has evolved over the last four decades into a specialized part of mathematical physics. The applications of the mathematical models for rogue events is directly relevant to technology development for prediction of rogue ocean waves, and for signal processing in quantum units. In this manuscript, a comprehensive view of the most recent development in conventional methods for representing rogue waves is carried out, along with discussion of the devised and forms and solutions. The standard nonlinear Schrödinger equation, the Hirota equation, the MMT equation and further to other models are discussed, and their properties highlighted. This review shows that the most recent advancement in modeling rogue waves give models which can be used to establish methods for prediction of rogue waves at open seas, which is important for the safety and activity of marine vessels and installations. The study further puts emphasis on the difference between the methods, and how the resulting models form a basis for representing rogue waves. This review has also a pedagogic component directed towards students and interested non-experts.
\end{abstract}

Keywords: rogue; wave; models, KdV, NLSE, non-local, ocean, optics

\section{Introduction}

Anomalous waves, or "rogue waves", represent a rare phenomenon at sea which occurs 19 approximately over 1500 times pr. year [1,2] and cause yearly millions of dollars of loss of cargo and loss of lives [3] Rogue waves are abnormally elevated waves, with a 2-3X height of the average wave normal and with unusually steep shapes [4,5]. Rogue waves were recorded for the first time in 1995 during a winter storm in the North Sea, when the "New Years Wave" hit the Draupner platform with a wave height of 27 meters and 2.25X the average wave height [4]. The laser-installation on the deck, which regularly records the elevation of the platform over the sea bed, registered the solitary giant wave with its $15.4 \mathrm{~m}$ elevation above and 11,6 meter below the zero-level [4]. The shape of the wave was symmetrical (Fig 1) with a Gaussian-bell shape and with a particular narrow wavelength. This shape and behavior of anomalous waves is conserved across several observations made in the last 25 years, including the rogue wave that hit the North Alwyn platform in November 1997 [6], the Gorm platform in 1984 [6] and from Storm 172 on the North Alwyn field 100 miles east of Shetland [5]. The latter was particularly unusual, with a height 3.19X the average (Fig 1). 

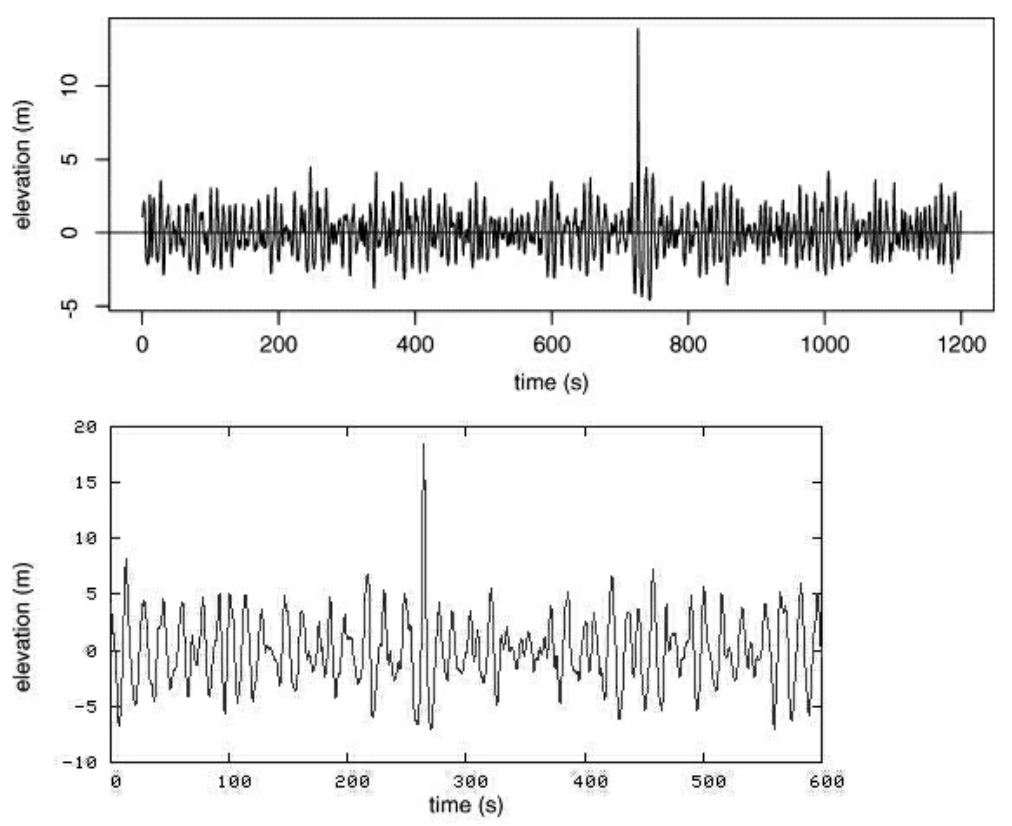

Figure 1. The laser-readings of the most extreme rogue wave registered (top), which hit the the North Alwyn platform east of Shetland [5], reaching 18.04 meters and a ratio of 3.19X with surrounding waves. Bottom: The New Years wave registered in 1995 on the Draupner platform (top) in the North Sea.

Rogue waves are known to have sunk over 20 supercarriers since 1970 [6] and carry a force of 16-20 times (100 metric ton $/ \mathrm{m}^{2}$ ) that of a 12 meter wave, and can easily break ship structures which are designed to withstand far lower impact forces $\left(6\right.$ metric ton $\left./ \mathrm{m}^{2}\right)$ [3]. Rogue waves are an eminent threat to shipping and naval activities, and increase in prevalence with climate-change weather patterns [7] In this context, the insurance sector has searched for new models for predicting rogue waves and for fortifying naval structures [3], as both off-shore installations, shipping and also cruise-ships have been increasingly exposed to rogue waves in the last decades $[3,6]$. This development has also sparked the project "Max Wave" [2] which has contributed with new models and algorithms for predicting rogue waves by the use of satellite observation data. Rogue waves occur also in optical systems [8] in the atmosphere [9] and in plasma [10].

Earlier mathematical models and derived algorithms that were used to predict wave patterns were originally developed by using the linear Gaussian random model, and rogue phenomena at sea were largely disregarded as superstition. The linear Gaussian model is essentially a superposition of elementary waves and predicts the occurrence of a rogue event at a very low probability. This low probability is however incorrect accounting for the laser-readings made in the last 2 decades at off-shor installations. Non-linear models which show a better agreement with the frequency of rogue events at sea, are therefore gradually replacing the Gaussian model used in the insurance industry. Non-linear models have been studied by several groups, and include the modified non-linear Schrödinger Equation (NLSE) [6], the Peregrine soliton model [11] the Levi-Civita and Nekrasov models [12,13], the Davey-Stewartson model [14], the fourth order partial differential equation of Kadomtsev-Petviashvili, the one-dimensional Korteweg-de Vries equation for shallow water surfaces, the second-order Zakharov partial differential equation [15], and the fully nonlinear potential equations. Other systems have recently been developed, and are here reviewed in detail given their relevance to rogue wave ocean phenomena, including the inhomogenous non-linear Schrödinger equation [16] the Akhmediev model [17-20], and the recent models developed by Cousins and Sapsis [21-23]. 


\section{The non-linear Schrödinger equation in prediction of rogue-waves}

Rogue waves occur both in oceans as well as in optical systems [11] and possibly in other wave-systems. A series of new classes of waves have been discovered in non-linear optics in recent years. One of these new types are the self-similar pulses [24-27]. Self-similar pulses are wave-amplitudes measured in fiber amplifiers [28], which experience an optical gain together with a Kerr-nonlinearity (Fig 2).

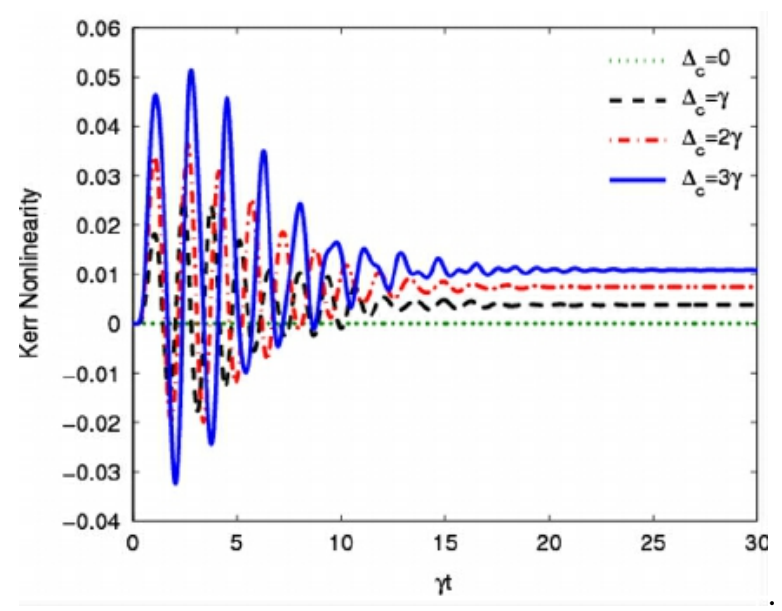

Figure 2. Observed Kerr-Nonlinearity in a crystal exposed to a magnetic field. [29]

During the induction of the self-similar impulse in the solid, a fluid or any wave-carrying medium, the shape of the resulting rogue wave no longer depends on the shape or duration of the seed pulses, but depends only on the seed pulse energy (chirping). This creates a large effect on the amplitude, which is largely independent on the initial conditions of the wave pattern. This event, or formation of a rogue component in the wave-train, has also been observed in ocean wave systems [30] and has attracted various groups to develop prediciton methods using the variations of the non-linear Schrödinger equation (NLSE) [16,24,28,31,32]. One group in particular developed the variable coefficient inhomogenous nonlinear Schrödinger equation (vci-NLSE) for optical signals [16]:

$$
i \psi_{x}+\frac{1}{2} \beta(x) \psi_{t t}+\chi(x)|\psi|^{2} \psi+\alpha(x) t^{2} \psi=i \gamma(x) \psi,
$$

which derives from the Zakharov equation [33]. Here $\psi(t, x)$ is the complex function for the electrical (wave) field and $\mathrm{x}$ and $t$ are respectively the propagation distance function and retarded time function. The parameter $\alpha(x)$ defines the normalized loss rate and the function $\alpha(x) t^{2}$ accounts for the chirping effects (which indicates that the initial chirping parameter is the square of the normalized growth rate). The parameter $\beta(x)$ defines the group-velocity dispersion (i.e. for an entire wave-train), while $\chi(x)$ defines non-linearity parameters, and $\gamma(x)$ defines loss or gain effects of the wave-signal. This equation is adaptable both for oceanic waves, as well as for optical non-linear wave guides. Equation (1) is essentially the same as the generalized Gross-Pitaevskii equation with the harmonic oscillator potentials in the Bose-Einstein condensates [34] and can be solved by applying the similarity transformation [35] by replacing $\psi(t, x)$ in equation (1) with:

$$
\psi(t, x)=\rho(x) \Psi(T, X) e^{i \phi(t, x)},
$$

where $\rho(x)$ is the amplitude, and $\mathrm{X}$ and T represent the differential functions describing original propagation distance and the similarity variable while $\phi(t, x)$ is the linear variable function of the exponential term, which all must be considered well to avoid singularity of the system $\psi(t, x)$ [16]. The similarity transformation gives: 


$$
i \Psi_{Z}+\frac{1}{2} \Psi_{T T}+|\Psi|^{2} \Psi=0,
$$

which is the standard non-linear Schrödinger equation.

The transformation and integrability conditions derived by [16] show that the factors of the wave system, such as effective wave propagation, distance, central position amplitude, the width and phase of the pulse are ultimately dependent on the group velocity dispersion and on the non-linearity parameters of the system $(\alpha, \beta, \gamma, \chi)$. The "self-similar" solution found in the process of the transformation of the variable coefficient inhomogenous nonlinear Schrödinger equation into the standard nonlinear Schrödinger equation can ultimately be controlled under dispersion and non-linearity management [16]. Once transformed from the iNLSE, the solutions to the NLSE are derived by the derivation of polynomial conjugates to the root exponential function. This process is reviewed in detail here from the studies by [18].

\subsection{The solutions to the NLSE}

The NLSE equation has been solved by various groups, including [15,18,35-37]. Following one of the most recent works by $[17,18]$ in particular, the steps for deriving exact solutions to the NLSE are defined by identifying rational solutions [17] for the homogenous nonlinear system in eqn. (3) by using the Darboux transformation [38]. This method is often used to derive rational solutions for non-linear systems and is adaptable to both to specific optical rogue waves as well as ocean rogue waves, when represented by the NLSE. The main definition of a rogue event is that the wave "appears from nowhere and vanish without a trace", which is feature closely related to the behavior of solitons, which are independent waves that self-propagate and exit a collision unchanged. The origin of solitons arises from the first observation of a single solitary wave in the North Sea, made in 1834 by J. S. Russell, who later reproduced the solitary wave in a tank. Since then, solitons have been mainly studied in optical systems, and are represented as solutions to several types of nonlinear PDEs, including the NLSE, the Korteweg de Vries equation and the Sine-Gordon equation. This type of rogue behavior, is described by the rational solutions derived from the NLSE [18], which describe an induction of a system instability to the top of a plane wave amplitude, which is transferred to the highest amplitude and then decays exponentially towards zero [17]. This behaviour is represented by Ma-solitons and by Akhmediev breathers or "Akhmediev solitons" [17,18,39-41]. The difference between these two soliton models lies in the initial conditions, where the Ma-solitons originates from the initial conditions while the Akhmediev solitons arise during evolution of the system given by modulation instability $[39,42,43]$.

When solving the NLSE according to the Akhmediev scheme [18], their method describes the modeled envelope function $(\psi)$ as a solution ranked into an order of hierarchy, starting from first, and progressing to the second, third or fourth order [18]. The difference between each order is the increasing amplitude of the rogue wave (first order -lowest amplitude, fourth order sharpest peak and highest amplitude). The envelope function $\psi$ is expressed as a ratio of polynomials multiplied to the complex exponential root function, $e^{i x}$. The polynomials, which are given by functions of variable $x$ and $t$, are solved by performing the Darboux transformation on the NLSE system [18]. Akhmediev and colleagues furthermore apply a compatibility-check between the root function $e^{i x}$ and the reference-state for two specified column matrix elements, which define initial conditions for the NLSE. These matrix elements (vectors) are given specifically by Akhmediev and colleagues [18] as two differential equations:

$$
\begin{gathered}
r_{x}=i l^{2} r+i l \psi^{*} s-i l 2|\psi| 2 r+\frac{1}{2} \psi^{*} s \\
s_{x}=i l^{2} s+i l \psi r-\frac{1}{2} \psi t r+\frac{i}{2}|\psi| 2 s
\end{gathered}
$$


which are split into real and imaginary parts, before being simplified and solved to fit into the modified Darboux scheme $[18,38]$ to give the two linear differential forms:

$$
\begin{aligned}
& r_{l}(x, t)=\sqrt{2}\left[x t-\frac{1}{2}+i x\right] e^{-i x / 2} \\
& s_{l}(x, t)=\sqrt{2}\left[x-i\left(t+\frac{1}{2}\right)\right] e^{-i x / 2}
\end{aligned}
$$

Where the two vectors (6), (7) are used in the Darboux scheme to find $\psi j$, where $j$ is the order of hierarchy. The general solution to the NLSE, derived from this scheme [18] is given by the following general form (for any order in the hierarchy):

$$
\psi_{j}(x, t)=\left[(-1)^{j}+\frac{G_{j}(x, t)+i x H_{j}(x, t)}{D_{j}(x, t)}\right] e^{i x}
$$

where $G, H$ and $D$ are the polynomials of the two variables $x$ and $t$ (mentioned above). The first order-solution [18] has the following polynomials: $\mathrm{G}=1, \mathrm{H}=2$ and $D=1+4 t^{2}+4 x^{2}$ which give the following envelope function (shown in Fig 3):

$$
\psi_{l}=\left[1-4 \frac{1+2 i x}{1+4 t^{2}+4 x^{2}}\right] e^{i x}
$$

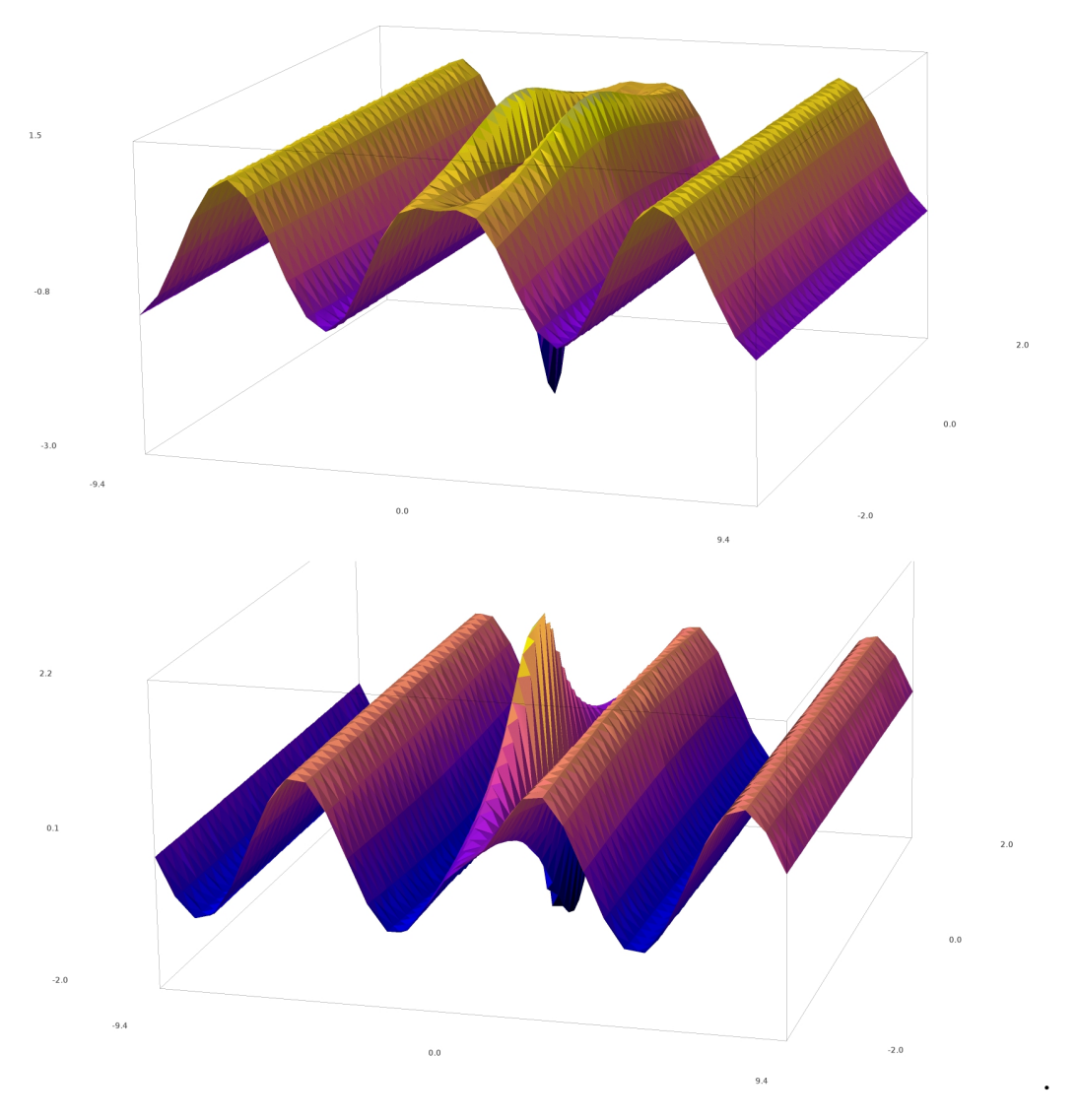

Figure 3. The plot of $\psi_{1}$, the first-order solution to the standard NLSE [18]. Real and imaginary part shown respectively above and below. Plotted with SageMATH [44,45].

For the second-order solution, [18] identify the vectors $\mathrm{r} 2$ and s2 by solving the equations (4) and (5) by using the form of $\psi$ given in (8). This gives the second-order solution: 


$$
\psi_{2}(x, t)=\left[(-1)^{2}+\frac{-2 t^{4}-12 t^{2} x^{2}+i x\left(8 t^{2} x^{2}+2 t^{2}-4 x^{4}-2 x^{2}+\frac{15}{4}\right)-3 t^{2}-10 x^{4}-9 x^{2}+\frac{3}{8}}{\frac{16 t^{6}}{3}+13 t^{2}+\frac{16 x^{6}}{3}+36 x^{4}+33 x^{2}+\frac{3}{4}}\right] e^{i x}
$$

which is shown in figure 4 . The third and fourth order rational solutions are furthermore calculated and given in [18].

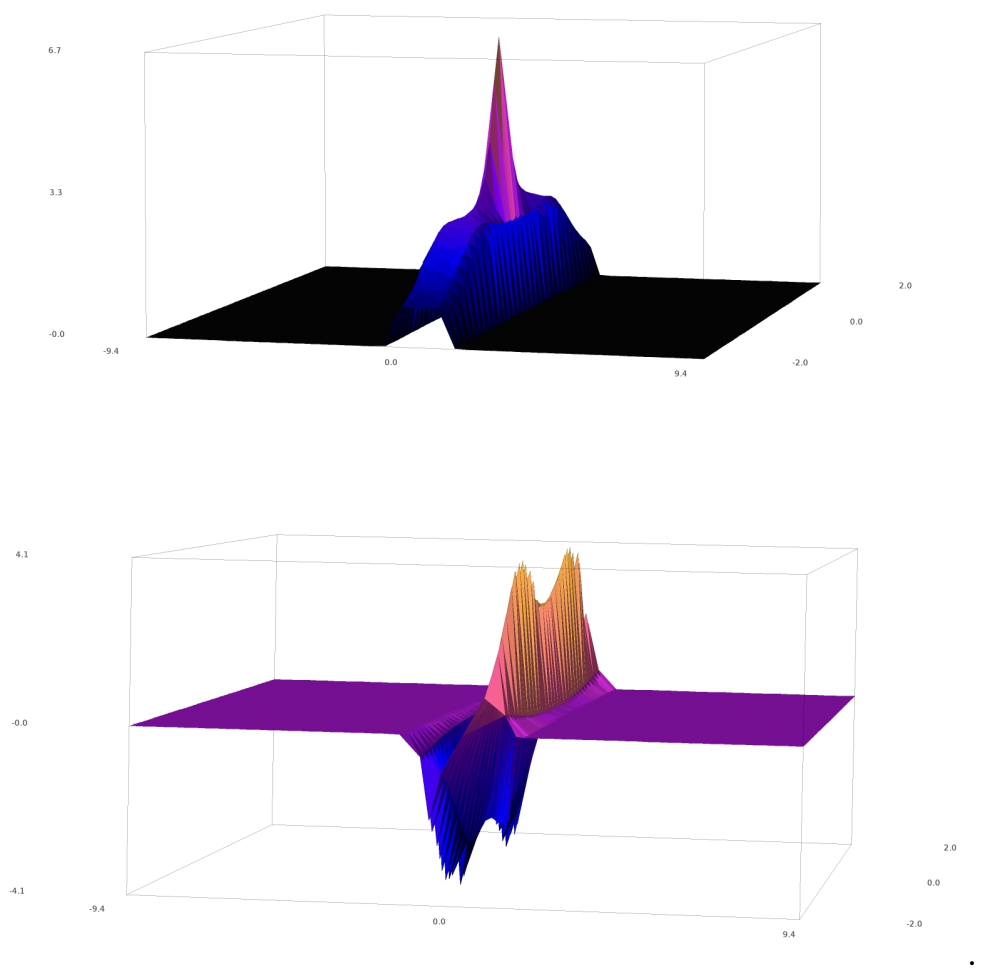

Figure 4. The plot of $\psi_{2}$, the second-order solution to the standard NLSE [18]. Real and imaginary part shown respectively above and below. Plotted with SageMATH [44,45].

The same hierarchy-dependency is given in the approach by [16], for the transformed vci-NLSE, who define the general solutions for the NLSE in the hierarchical $n$-th order given by:

$$
\psi_{n}=\frac{1}{W} \sqrt{\frac{\beta}{\chi}}\left[(-1)^{-1}+\frac{G_{n}+i\left(Z-Z_{0}\right) H_{n}}{F_{n}} \times e^{i\left[\left(1-\frac{v^{2}}{2}\right)\left(Z-Z_{0}\right)+v T+\phi\right]}\right.
$$

where each factor is given for the first and second order rational solutions [16]. Similarly to the hierarchy solutions of Akhmediev [18], the increasing order gives higher and higher rogue waves, compared to their surrounding waves. The first and second order rational solutions given in [16] reflect respectively a $3 \mathrm{X}$ and $5 \mathrm{X}$ rogue wave height, compared to the surrounding waves. For plots of these, refer to [16].

The similarity between (11) and (8) is striking, and both retain the basic form of a complex polynomial multiplied by a complex exponential root function giving soliton solutions. The root functions of (8) and (11) are shown in their generic form in Fig 5, which depicts the distinction between the seed pulse for the regular NLSE and the vci-NLSE, as studied respectively by $[16,18]$ for the rogue wave problem. 

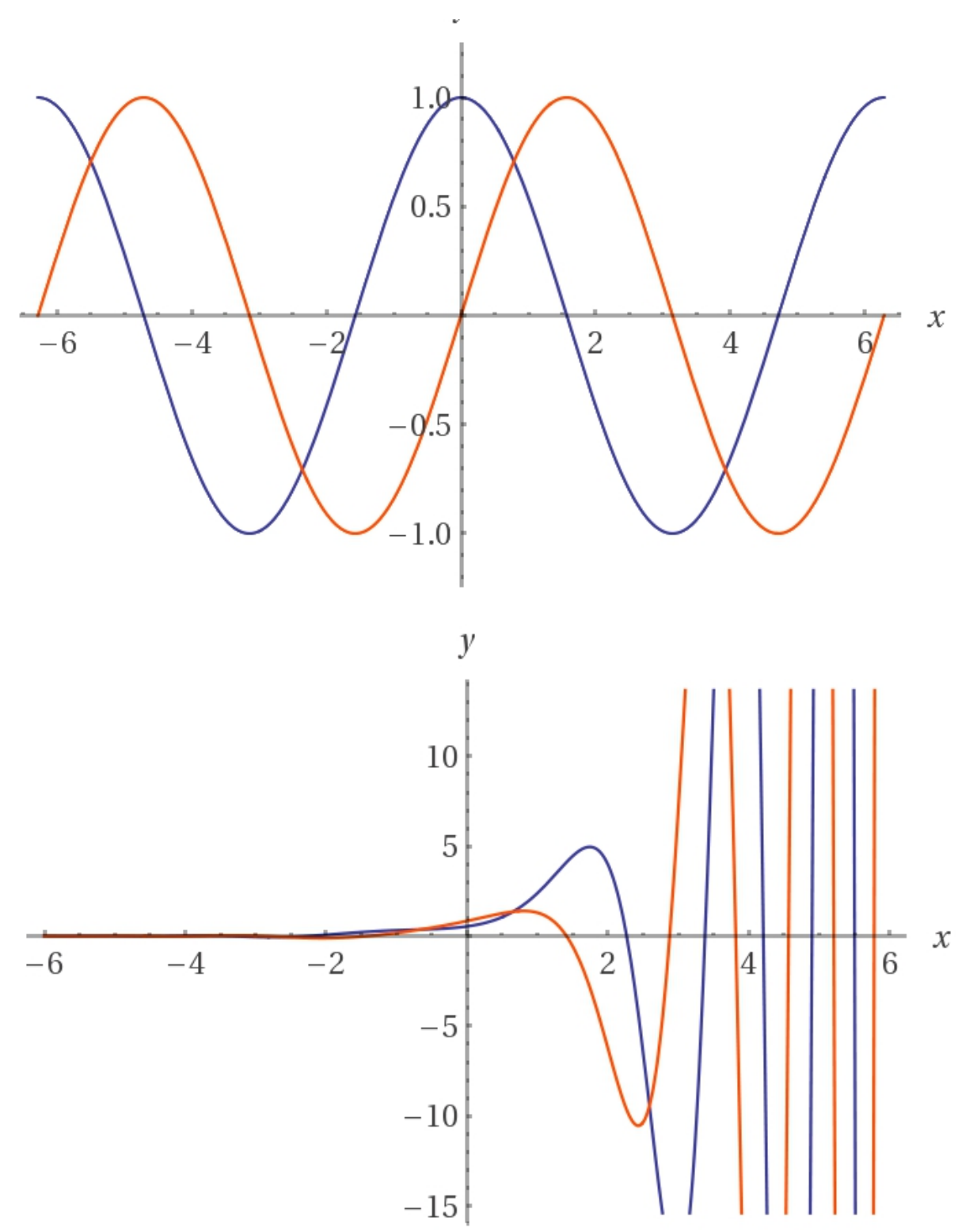

Figure 5. The root functions for the standard NLSE and the inhomogenous variable coefficient NLSE. Top: The seed impulse used in the solutions to the standard NLSE, $e^{i x}$ [18]. Bottom: A generic form of the seed impulse used in the solutions of the inhomogenous variable coefficient NLSE [16] $\left(f(x)=e^{i\left(1-x^{2} / 2\right)+x}\right.$. Real part (Blue) and imaginary part (Red).

The root function for the vci-NLSE (Fig 5) shows its specific pattern of wave accumulation, which is similar to the formation of wave packets. This pattern is conserved with the physical behavior of rogue wave formation, where the rogue wave forms during a focusing phase [46]. Other approaches to solve the NLSE have been given by [32], who used the inverse scattering method of transformation, which is a generalization of the Fourier analysis. Their solutions differ from the methods discussed above, and are periodic and are ascribed by a complex envelope function for the deep water train with added higher-order terms from the perturbation procedure [32] One of the solutions are shown in figure 6, which shows the following variant of the Osborne models:

$$
\psi=\frac{\cos (\sqrt{2} x) \operatorname{sech}(\sqrt{2} t)+i \sqrt{2} \tanh (2 t)}{\sqrt{2}-\cos (\sqrt{2} x) \operatorname{sech}(\sqrt{2} t)} e^{2 i t}
$$


which is a periodic function in space, derived from the general form given in [32], shown in Figure 6.

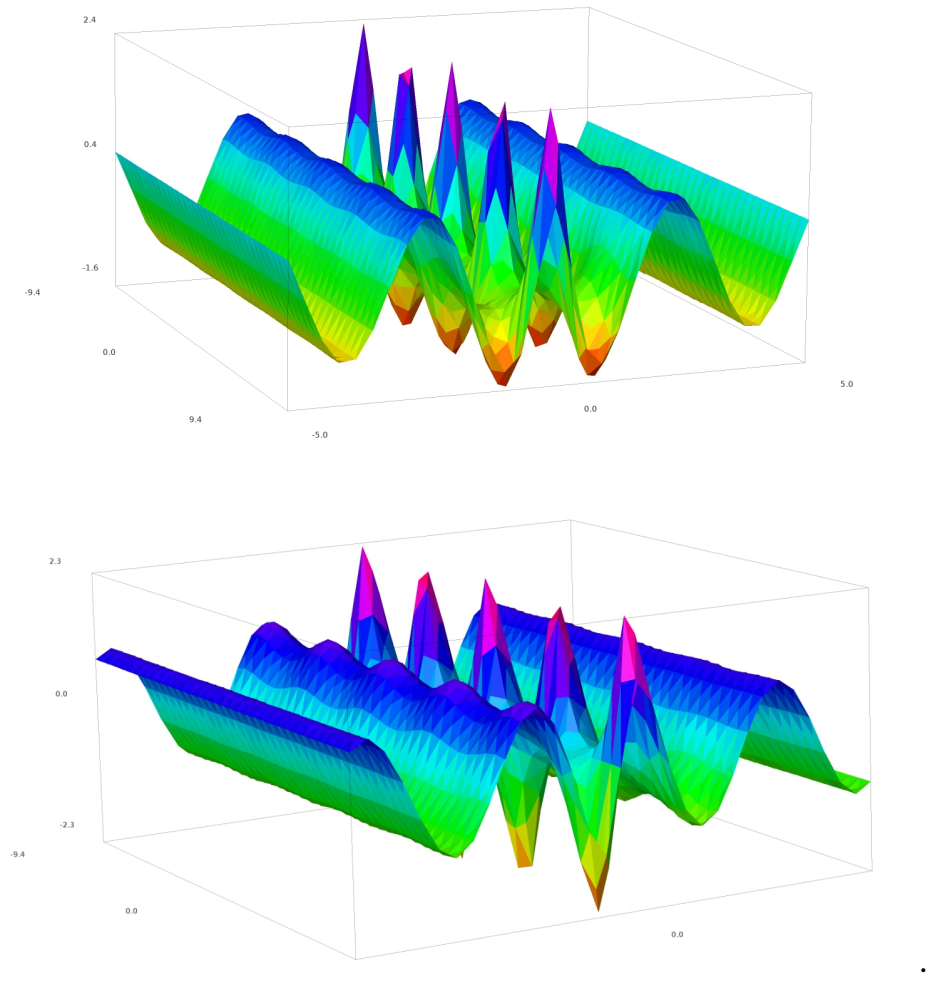

Figure 6. The selected wavefunction from the Osborne models [32]. Top: Real part; Bottom: Imaginary part. Plotted with SAGEMATH $[44,45]$

The disadvantage of this system, compared to single-peak models derived from [18] lies in their periodicity and multiple peaks, while the rational solutions behind the single peak models of $[17,18]$ are the first in general to serve as prototypes for rogue waves.

\section{The Korteweg de Vries equation}

Wave systems defined by higher order nonlinear PDEs, such as (2), can be solved also by the bilinearization technique [47]. This technique involves the step of transforming the differential equation into a more tractable form by replacing the unknown time- and position-dependent envelope function with a new form [47]. After this replacement has been performed, the bilinearization technique applies Hirota bilinear operators for a modified Bäcklund transformation technique [48], which assists in rewriting the original PDE into a simplified PDE composed of bilinear operators, from where exact soliton solutions can be identified. The most suitable example [47] for the application of the bilinearization technique is on the Korteweg de Vries (KdV) equation:

$$
\Psi_{t}+6 \Psi \Psi_{x}+\Psi_{x x x}=0
$$

where the boundary conditions are that $\psi \rightarrow 0$ as $|x| \rightarrow \infty$. The real wavefunction is differentiated according to the spatial and temporal dimensions as denoted. In the bilinearization technique, a transformation of the wavefunction to another form is the first step, where an ideal steady-state form is proposed [47] to:

$$
\psi(x, t)=\left(p^{2} / 2\right) \operatorname{sech}^{2}(\eta / 2),
$$

where 


$$
\eta=p x-p^{3} t+\eta_{0}
$$

and $\eta_{0}$ and $p$ are arbitrary constants. By the bilinearization technique [47], one can rewrite (14) to the form:

$$
\psi(x, t)=2 p^{2}\left(e^{\eta / 2}+e^{-\eta / 2}\right)
$$

which is converted to its functional form:

$$
\psi(x, t)=2 \frac{\partial^{2} \ln (f(x, t)}{\partial x^{2}}
$$

with $\mathrm{f}(\mathrm{x})=1+e^{\eta}$.

The bilinearization technique [47] substitutes (17) into the original KdV equation (13) and integrates it with respect to $\mathrm{x}$ :

$$
f_{x t} f-f_{x} f_{t}+f_{x x x x} f-4 f_{x x x} f_{x}+3\left(f_{x x}\right)^{2}=0
$$

which is the original version of the bilinearized variant of the Korteweg de Vries equation (13) as derived by [48] The solution to (18), $\mathrm{f}(\mathrm{x})=1+e^{\eta}$, is defined as a more fundamental quantity than $\psi$ in eqn. (16) for the structure of the original nonlinear PDE in eqn. (13). In the method of bilinearization, the Hirota bilinear operators are introduced. These are defined by the following definition [48]:

$$
D_{t}^{n} D_{x}^{m} a \cdot b=\left.\left(\partial / \partial t-\partial / \partial t^{\prime}\right)^{n}\left(\partial / \partial t-\partial / \partial t^{\prime}\right)^{m} a(x, t) b\left(x^{\prime}, t^{\prime}\right)\right|_{x=x^{\prime}, t^{\prime}=t}
$$

with $m$ and $n$ being arbitrary positive integers. At this stage, the converted form of the KdV equation (18) is rewritten as a PDE composed of Hirota operators:

$$
D_{x}\left(D_{t}+D_{x}^{3}\right) f \cdot f=0,
$$

which is a simplified form for the identification of exact solutions using the Bäcklund transformation for the original nonlinear PDE (13). The exact solution structure for the type of Hirota-operator based PDE form (20) of the KdV equation (13) is given by:

$$
\Psi=1+\epsilon\left(e^{\eta_{1}}+e^{\eta_{2}}\right)-\epsilon^{2} \frac{F\left(\Omega_{1}-\Omega_{2}, p_{1}-p_{2}\right)}{F\left(\Omega_{1}+\Omega_{2}, p_{1}+p_{2}\right)} e^{\eta_{1}+\eta_{2}}
$$

which represents the two-solition solution to the original KdV equation (13). $\eta_{1}$ and $\eta_{2}$ are the functions with the independent variables $x$ and $t$ as given in (14) for each of the solitons, and $\Omega_{1}=-p_{1}^{3}$ and $\Omega_{2}=-p_{2}^{3}$, following the same definition for (14) for each soliton. $\eta$ represent the perturbation [47]. The KdV equation (13) has also been solved by Matveev by identifying positon solutions [49], which exert the same behavior as solitons, such as conserved shape after collision, and elastic collision behavior. The positon differs from the soliton in that it has an infinite energy, and is therefore not a strong model for oceanic or optical rogue waves. Positons have however a tendency to represent smoother solutions than solitons to the $\mathrm{KdV}$ equation, and can have very high peaks compared to the wave normal. The KdW equation has also been solved by a nonlinear Fourier method [50,51] which is represented by a superposition of nonlinear oscillatory modes of the wave-spectrum. This model by Osborne $[50,51]$ has a capacity to include a large number of non-linear oscillatory patterns, also known as multi-quasi-cnoidal waves, which are used to form the rogue wave by superposition in constructive phases. These solutions to the original KdV equation (13) include several solitons, depending on the number of degrees of freedom selected for the numerical simulation of the KdV equation. This yields a 3D wave complex composed of solitons and radiation components in the simulated wavetrain [50]. 


\section{The extended Dysthe equation}

In 1979, Dysthe [52] developed a modification of the perturbation-based NLSE by adding an additional term to the third-order perturbation variant originally developed by Higgins [53]. Dysthe's method gave an NLSE variant, known as the extended Dysthe equation, which showed to have a better agreement with the mean flow response to non-uniformities in deep-water waves. The extended Dysthe equation is given by:

$$
\frac{i}{k} \psi_{x y y}+\psi_{y y}+2 i k \psi_{x}+2 \psi_{z}=o \epsilon^{4}
$$

where the inhomogenous component is the fourth-order perturbation defined by Dysthe [52]. Dysthe transformed this equation to standard NLSE by using dimensionless variables, and added the following perturbation to the general solution:

$$
\psi=c_{0}(1+\alpha) e^{i\left(\theta^{\prime}-\frac{1}{2} i c_{0}^{2} t\right)}
$$

where $\alpha$ and $\theta$ are small real perturbations of the amplitude and phase respectively. After insertion of (23) in the dimensionless form of (22) and linearizing, Dysthe obtained a simplified system of two PDEs, where the respective plane-wave solutions are in the form:

$$
\left(\begin{array}{c}
\alpha^{\prime} \\
\theta^{\prime}
\end{array}\right) \propto e^{i(\lambda x+\mu y-\Omega t)}
$$

and

$$
\bar{\phi} \propto e^{[K z+i(\lambda x+\mu y-\Omega t)]}
$$

where $\mathrm{K}=\sqrt{\left(\lambda^{2}+\mu^{2}\right)}$ and $\lambda, \mu$ and $\Omega$ are selected parameters which satisfy a set of dispersion relations given by Dysthe [52].

The stability of the solutions derived by Dysthe show that the Dysthe equation represents a more realistic model than the NLSE, given that it does not predicts a maximum growth rate for all wavevectors, but only for some wavevectors only. This displays that the fourth-order perturbation term added to the NLSE gives a considerable improvement to the results relating to the stability of the finite amplitude wave. It is particularly the first derivative by the transformed variables in the $x$ and $z$ dimensions in eqn. (22) which contributes to the excellent results of Dysthe. Dysthe and Trulsen [54,55] further developed this equation by including up to the fifth-order of the derivative of the wave amplitude describing the linear dispersive terms, and simulated successfully [56] the New Year's wave [4] using the extended Dysthe equation [52,56].

\section{The MMT model}

The MMT equation is a one-dimensional nonlinear dispersion equation which was originally proposed by Majda, McLaughlin and Tabak [57]. The MMT equation gives soliton-like solutions which have been analyzed in detail by Zhakarov [58-60] and gives four-wave resonant interaction between waves, which, when coupled with large scale forces and small-scale damping, yields a family of solutions which exhibit direct and inverse cascades [21]. The MMT equation is given by:

$$
i \psi_{t}=\left|\partial_{x}\right|^{\alpha}+\lambda\left|\partial_{x}\right|^{\beta / 4}\left(\left.\left.|| \partial_{x}\right|^{\beta / 4} \psi\right|^{2}\left|\partial_{x}\right|^{\beta / 4} \psi\right)+i D \psi
$$

where $\psi$ is a complex scalar and $\left|\partial_{x}\right|^{\alpha}$ is the pseudodifferential operator defined on the real axis through the Fourier transform:

$$
\left|\partial_{x}\right|^{\alpha} \psi(k)=|k|^{\alpha} \widehat{\psi}(k)
$$


The last term in (26) is the dissipation term, which is tuned to fit ocean waves through the Laplacian operator, $\mathrm{D} \psi$, defined in the Fourier space:

$$
\widehat{D \psi}(k)=\left\{\begin{array}{lc}
-\left(|k|-k^{*}\right)^{2} \widehat{\psi}(k) & |k|>k^{*} \\
0 & |k| \leqslant k^{*}
\end{array}\right.
$$

This dissipation term, used by [21] is similar to other dissipation models used by Komen and colleagues [61], who have developed concrete models for simulating large wave groups with focusing and defocusing effects. $\lambda$ is the nonlinearity coefficient and corresponds to the focusing phase when $<0$, and to the defocusing phase when $>0$. The MMT equation (26) differs from the standard NLSE by that its family of solutions develop in a more exponential pattern, rather then the Gaussian-bell shaped pattern observed for the solutions for the NLSE [21]. The interesting aspect of this pattern of the spectrum of solutions of the MMT equation is in the mode of formation of the rogue wave, where there energy is transferred from and to the surrounding waves. The solutions are in other words induced by the intermittent formation from the localized rogue event arising out from the regular Gaussian background and collapsing into the surrounding waves. The energy of the rogue wave is transferred to the surroundings and experiences a complete zero-point state, merging completely in the background [21].

The MMT model shows also the formation of quasisolitons which appear in triple-wave packets, as modelled by Zakharov and Pushkarev [58] and differ from regular solitons in that they radiate the energy backwards towards the preceding amplitudes. This behavior of the solutions may be particularly compatible with the simulation of rogue wave events occurring in regions with strong counter-wind currents, such as in the Aghulas-current [62] or in the regions of the Irish sea [2], which are heavily populated by rogue events, on the passage of the warm waters of the Gulf stream when encountering the frequent low-pressure systems over the Irish sea with counter-wave winds. The quasibreathers or quasisolitons [58], have the root function similar to the Dysthe-type solutions given in (23). Zakharov and Pushkarev [58] approach the solutions in the form:

$$
\psi(t)=e^{i(\Omega-k V) t} \phi_{k}
$$

where $\Omega$ and $\mathrm{V}$ are constants $(\Omega<0$ and $V>0)$, and $\mathrm{k}$ is the wavenumber, which is an approximate solution to the soliton-like solution for the MMT model. In this approximation, [58] give $\phi \mathrm{k}$ the following form:

$$
\phi_{k}=\lambda \frac{\int T_{1234} \phi_{1}^{*} \phi_{2} \phi_{3} \delta\left(k+k_{1}-k_{2}-k_{3}\right) d k_{1} d k_{2} d k_{3}}{-\Omega+k V-|k|^{\alpha}},
$$

which represent a form which gives quasi-soliton solutions [63] to the MMT equation. This form of the solutions to the MMT equation radiate energy backwards to the proceeding amplitudes, and represent therefore a energy focusing which is rather un-similar from the focusing effects modeled by others for rogue patterns (vide supra). It is interesting to note that backward radiation plays also a central role for the dynamics of the quasi-solitons, and not only for their energy-accumulation profile. Using the MMT model, Zakharov and Pushkarev [58] developed also a model for collapses of the rogue event, by using self-similar solutions, and model the formation of the wave wedge in the appearing and vanishing state, given by a Fourier-space distribution of the wave-function. Zakharov and Pushkarev [58] have also used the MMT model to develop turbulence-based solution for the localized rogue event, using the initial condition in the form of a NLSE soliton:

$$
\psi(x, 0)=\frac{q}{2 k_{m}^{9 / 4}} \frac{e^{i k_{m} x}}{\cosh (q x)},
$$

which shows a conserved action and momentum, and an "inner turbulence" localized both in the real and Fourier spaces of the solutions to the modeled envelope function. This "intrinsic turbulence" 
is described by the authors in affecting the form of its wave-spectra, which is irregular and with a stochastic behavior [58]. This model of the rogue wave shows quasi-periodic oscillations with slowly diminishing amplitudes over time - caused by the destruction of rogue wave by the surrounding interference, which the authors denoted as a quasi-breather.

\section{The Hirota equation}

Multisolitons and breathers for rogue waves have been also successfully modeled [64] by applying the Darboux transformation on the Hirota equation [48]. In their approach, Tao and He [64] developed the Lax pair on the Hirota equation, by using the AKNS [65] procedure to get the Lax pair with the spectral parameters of the Hirota equation given below:

$$
i \psi_{t}+\alpha\left(2|\psi|^{2} \psi+\psi_{x x}\right)+i \beta\left(\psi_{x x x}+6|\psi|^{2} \psi_{x}=0\right.
$$

where the Lax pair is expressed as:

$$
\phi_{x}=M \phi, \phi_{t}=N \phi
$$

giving rise to the extended matrix representation of the operators in the Hirota equation as given by Tao and He [64]. Tao and He further applied the Darboux transformation [38] on the Lax-represented system by using the simple gauge transformation for spectral problems,

$$
\phi^{[1]}=T \phi
$$

where $\mathrm{T}$ is the polynomial applied on the parameter $\lambda$ given in the Lax pair, and $\phi$ is the seed function. Tao and He [64] argue however that regular seed solution $\phi=e^{i x}$ as described above in the previous sections is too special, and makes the rogue wave model not universal enough. Tao and He develop therefore a different seed function compared to for instance Akhmediev and colleagues $[18,39]$ and develop a more extended form of the seed function by starting from a zero seed solution and a periodic seed solution to construct the complete solutions for the breathers and solitons. At zero seed and with the parameter $\lambda$ from the Lax pair they set the following Hermitian seed pair:

$$
\phi=e^{-i(\tilde{\xi}+i \eta) x-\left(4 \beta i(\xi+i \eta)^{3}+2 \alpha i(\tilde{\xi}+i \eta)^{2} t\right.}
$$

and

$$
\phi^{*}=e^{i(\xi+i \eta) x+\left(4 \beta i(\tilde{\xi}+i \eta)^{3}+2 \alpha i(\tilde{\xi}+i \eta)^{2} t\right.}
$$

back in the Darboux Transformation to get the 1-soliton solution:

$$
\psi_{\text {soliton }}^{[1]}=2 \eta e^{2 i\left(-i \xi x-4 \beta \xi^{3} t-2 \alpha \xi^{2} t+12 \beta \xi^{2} \eta^{2} t+2 \alpha \eta^{2} t\right)} \times \operatorname{sech}\left(-2 \eta x-24 \beta \eta^{2} t+8 \beta \eta^{3} t-8 \alpha \eta \xi t\right) .
$$

Tao and He [64] further report the model for the 2-soliton solution, and finally give the form of the 1-soliton breather solution:

$$
\psi_{\text {breather }}^{[1]}=e^{i \phi}\left[c-\frac{2 \eta\left[\eta \cosh \left(2 d_{2}\right)-i \sigma \sinh \left(2 d_{2}\right)-c \cos \left(2 d_{1}\right]\right.}{c \cosh \left(2 d_{2}\right)-\eta \cos \left(2 d_{1}\right)}\right],
$$

where $d_{1}, d_{2}, \sigma$ are given [64]. Tao and He finally construct the Rogue wave solutions to the original Hirota equation in (32) by Taylor expansion on the breather solutions in (38). The Taylor expansion is carried out at the $\eta$ variable of the breather solution (38) which is given in [64], and forms the general form of the first order rogue wave of the Hirota equation: 


$$
\psi_{\text {roguewave }}=k e^{i(-2 \xi x+\beta t)}\left(1-\frac{2 k_{1}+2 k_{2}+i k_{3} t}{k_{1}-k_{2}}\right)
$$

where the polynomials $k_{1}, k_{2}, k_{3}$ are given by Tao and He [64]. The rogue wave model resulting from this form is more general than the model given by Akhmediev and colleagues [66] on the Hirota equation. This difference is caused by the appearance of several parameters related to the eigenvalues of the Lax pairs, and gives however a possibility to tune more finely the model to experiments on rogue waves. This advantage of the model by Tao and He increases the ability to modulate the precision of reproducing a rogue wave model by calculations. Tao and He's method grants also the possibility in calculating higher order rogue wave solutions to the Hirota equation by determinant representation of the Darboux Transform, which was carried out in a subsequent work [67].

\section{The Ablowitz models: Non-local rogue waves}

Another critical method for modelling rogue waves was developed by Ablowitz and Musslimani [68-71] and uses nonlocal integrable models of the NLSE (1) and KdV (13) equations, where the resulting wave is derived by reverse space-time symmetry. The model, evolves by establishing integrability by an infinite number of constants of motion or an infinite number of conservation laws. By this, the method uses a compatible pair of linear equations (similar to the Lax pair in the Hirota equation in (33)) with the nonlinear integrable equation. The method by Ablowitz and Musslimani differs from the Hirota method in that the pair of linear equations represent the scattering problem and the evolution of the scattering data [68,71]. Furthermore, the method by Ablowitz and Musslimani is different from others in that it constructs an inverse scattering problem also known as a linear Riemann-Hilbert problem, which gives the solution to the nonlinear PDE with dependency on time. The approach by Ablowitz and Musslimani [68] starts by linearizing the equation:

$$
i q_{t}(x, t)=q_{x x}(x, t) \pm 2 q(x, t) q^{*}(-x, t) q(x, t),
$$

where one can immediately observe the existence of a Hermitian pair with reverse directional variables. This form, where reverse variables are used, defines the nonlocal property of the equation and has the advantage by that the equation remains invariant in time and space, after the complex conjugate is taken. Hence, the nonlocal equation is parity- and time- symmetric ( $\mathcal{P} \mathcal{T}$-symmetric), which prevents the equation from yielding different results by a self-induced potential.

An exemplary Lax pair is given in [68] as:

$$
\begin{gathered}
v_{x}=\left(\begin{array}{cc}
-i k & q(x, t) \\
r(x, t) & i k
\end{array}\right) v, \\
v_{t}=\left(\begin{array}{cc}
A & B \\
C & -A
\end{array}\right) v,
\end{gathered}
$$

where $v$ is the two-component vector and $k$ is a special parameter, and A and B are complex functions. Ablowitz uses at this step specific compatibility conditions [72] to transform the original PDE in (40) (i.e. $\psi_{x t}=\psi_{t x}$ ) and gains the simplified PDE pair:

$$
\begin{aligned}
& i q_{t}(x, t)=q_{x x}(x, t)-2 r(x, t) q^{2}(x, t), \\
& -i r_{t}(x, t)=r_{x x}(x, t)-2 q(x, t) r^{2}(x, t) .
\end{aligned}
$$

which yield the original form in eqn. (40). by using a specific symmetry reduction which defines the nonlocality: 


$$
r(x, t)=\mp q^{*}(-x, t) .
$$

This step is particularly characteristic to Ablowitz models [68-71], which of the new class of nonlocal integrable evolution equations with the nonlocal NLSE hierarchy are directly derived by. The aforementioned property of conserved quantities and conservation laws is also characteristic to Ablowitz models $[68,70]$. Here they define a set of eigenfunctions which obey specific boundary conditions [73]. The eigenfunctions are very similar to the seed functions used by other groups and when inserted in the Lax pair, yield a Riccati model of the conservation quantities. This yields the global conservation laws which are given in [68] by:

$$
\begin{gathered}
\mathcal{C}_{0}=\int_{-\infty}^{+\infty} q(x, t) q^{*}(-x, t) d x, \\
\mathcal{C}_{1}=\int_{-\infty}^{+\infty}\left[q_{x}(x, t) q^{*}(-x, t)+q(x, t) q_{x}^{*}(-x, t)\right] d x, \\
\mathcal{C}_{2}=\int_{-\infty}^{+\infty}\left[q_{x}(x, t) q_{x}^{*}(-x, t)+\sigma q^{2}(x, t) q^{* 2}(-x, t)\right] d x,
\end{gathered}
$$

which are real integrable Hamiltonians. Ablowitz and Musslimani [68] derive furthermore local conservation laws defined by the equations:

$$
\begin{gathered}
\partial_{t}\left[q(x, t) q^{*}(-x, t)\right]+i \partial_{x}\left[q(x, t) q_{x}^{*}(-x, t)+q^{*}(-x, t) q_{x}(x, t)\right]=0 \\
\partial_{t}\left[q(x, t) q_{x}^{*}(-x, t)\right]+i \partial_{x}\left[q_{x}^{*}(-x, t) q_{x}(x, t)+q(x, t) q_{x x}^{*}(-x, t)-\sigma q^{2}(x, t) q^{* 2}(-x, t)\right]=0
\end{gathered}
$$

which from they further develop the framework for the direct scattering problem and the inverse scattering problem, where the scattering data is given specific scattering matrices. The same symmetry is also in the problem of the potential and in the eigenfunction and leads naturally to the same symmetry relation in the scattering matrices, which are given by:

$$
N(x, k)=\Lambda M^{*}\left(-x,-k^{*}\right)
$$

and

$$
\bar{N}(x, k)=\Lambda^{-1} \bar{M} *\left(-x,-k^{*}\right),
$$

where $\Lambda$ is a $2 \times 2$ matrix with zeros in the diagonal and $1, \pm 1$ on the lower and upper diagonal respectively. For the inverse scattering problem, Ablowitz and Musslimani [68] account for the symmetry condition by considering the set of basis terms as a left scattering problem, and supplement these terms with the equivalent right-scattering problem, which from they formulate the Riemann-Hilbert problem and find the linear integral equations which govern the functions $\mathrm{M}$ and $\bar{M}$ in (48). These equations are given by:

$$
\begin{aligned}
& M(x, k)=\left(\begin{array}{l}
1 \\
0
\end{array}\right)+\sum_{l=1}^{J} \frac{\bar{B}_{l} e^{2 i \bar{k}_{l} x} \bar{M}\left(x, \bar{k}_{l}\right)}{k-\bar{k}_{l}}-\frac{1}{2 \pi i} \int_{-\infty}^{\infty} \frac{\bar{R}(\xi) e^{2 i \xi x} \bar{M}(x, \zeta)}{\zeta-(k+i 0)} d \zeta \\
& \bar{M}(x, k)=\left(\begin{array}{l}
0 \\
1
\end{array}\right)+\sum_{l=1}^{J} \frac{B_{l} e^{2 i k_{l} x} M\left(x, k_{l}\right)}{k-k_{l}}-\frac{1}{2 \pi i} \int_{-\infty}^{\infty} \frac{R(\xi) e^{2 i \tilde{x} x} M(x, \zeta)}{\zeta-(k+i 0)} d \zeta
\end{aligned}
$$

where $R(k)$ and $\bar{R}(x)$ are the reflection coefficients. The terms $B_{l}$ and $\bar{B}_{l}$ are the conservation law Hamiltonians applied symmetrically. From this stage, Ablowitz and Musslimani [68] derive a linear 
algebraic integral system of equations that solve the inverse problem for the eigenfunctions $\bar{M}(x, k)$ and $M(x, k)$.

The resulting soliton solutions of the Ablowitz model assume hence the form

$$
q(x)=-\frac{2(\eta+\bar{\eta}) e^{i \bar{\theta}_{1}} e^{-4 i \bar{\eta}_{1}^{2} t} e^{-2 \bar{\eta}_{1} x}}{1+e^{i\left(\theta_{1}+\bar{\theta}_{1}\right)} e^{4 i\left(\eta_{1}^{2}-\bar{\eta}_{1}^{2}\right) t} e^{-2\left(\eta_{1}+\bar{\eta}_{1}\right) x}}
$$

which represents a family of solutions defined by the four independent parameters which have a dynamic relationship with the time-variable and which gradually develop a singularity in a finite time period, $t_{s}$ at $\mathrm{x}=0$ where $t_{s}$ is given by:

$$
t_{s}=\frac{(2 n+1) \pi-\theta_{1}-\bar{\theta}_{1}}{4\left(\eta_{1}^{2}-\bar{\eta}_{1}^{2}\right)} .
$$

(53) is a critical form of the time-variable which distinguishes the method of Ablowitz [68-71,74] from other rogue waves models and adds a non-linear evolution of the rogue wave.

Solutions to the Ablowitz model (40) were also developed by Yang and Yang [75], who used the Darboux transformation method on the PDE coupled with the Bäcklund transformation on the potential functions, identifying three types of rogue waves from the Ablowitz picture. Yang and Yang expanded the solutions to polynomials using Schur polynomials [75]. This analysis of the Ablowitz model showed greater variation in the rogue waves compared to the regular NLSE, where the variations were represented by the terms in the denominator of the soliton solutions.

The parity-time symmetry potential of the Ablowitz equations has also been studied by $\mathrm{Yu}$ [76] very recently, who obtained discrete rogue wave solutions with three free parameters (refer to eqn. (52) for similarities). Yu studies in particular the effect that the dispersion of the parity-time symmetry has on the solutions, as well as the effect of the coefficients and the parameters. Yu [76] uses the Darboux transformation method in a similar fashion to Yang and Yang [75] to derive different forms of solitons with different height which are defined by two of the three free parameters in the solution ( $\eta$ and $\bar{\eta}$ in eqn. (52)). Yu [76] also assesses the stability of rogue waves over a specific period of time, and includes a modulation instability coefficient which allows the modelling of several discrete solutions which represent various stages of a rogue wave formation (appearing suddenly and disappearing suddenly), a property of rogue waves also reported by Akhmediev and colleagues [17-19]. Finally, Yu models rogue waves which appear rapidly and do not disappear. This latter model may be particularly relevant to describe rogue events during low-pressure systems at open sea, which have been reported in several cases to give stable rogue waves with long life-time (i.e. the rogue waves reported in the study by Munk [30]).

\section{Conclusions}

A review of various mathematical models for representing rogue waves has here been carried out to the maximum extent of including the most applied and conventional models. The overall review shows several common traits between the methods, and that new and emerging models allow for better modelling of rogue waves, by including several parameters associated with the evolution of the rogue waves, the duration, the height and other particular properties a rogue event can display when occuring in oceans, optical systems, or even in the atmosphere. The results shows also versatile structures of the older models (MMT and Dysthe), which can be furthermore adapted and studied numerically in upcoming papers, with emphasis on the inclusion of counter-wave winds, which frequently occur against the Gulf-current outside the Irish coast and on the Western Norwegian coast during North-East and North winds respectively. The purpose of this study was to collect and simplify the view of the models used to represent rogue events into pedagogically supported mathematical equations and arguments. This review can therefore be valuable as educational material and form a comprehensive description of the models for simulating and calculating the properties of rogue waves. 
Funding: This research received no funding.

Conflicts of Interest: The author declares no conflict of interest.'

\author{
Abbreviations \\ MMT Majda, McLaughlin and Tabak \\ AKNS Ablowitz, Kaup, Newell Segur
}

The following abbreviations are used in this manuscript:

\title{
References
}

1. Lehner, S.; Schulz-Stellenfleth, J.; Niedermeier, A.; Horstmann, J.; Rosenthal, W. Extreme waves detected by satellite borne synthetic aperture radar. ASME 2002 21st International Conference on Offshore Mechanics and Arctic Engineering. American Society of Mechanical Engineers, 2002, pp. 251-256.

2. Rosenthal, W.; Lehner, S. Rogue waves: Results of the MaxWave project. Journal of Offshore Mechanics and Arctic Engineering 2008, 130, 021006.

3. Didenkulova, I.; Slunyaev, A.; Pelinovsky, E.; Kharif, C. Freak waves in 2005. Natural Hazards and Earth System Science 2006, 6, 1007-1015.

4. Haver, S. A possible freak wave event measured at the Draupner Jacket January 1 1995. Rogue waves 2004 2004, pp. 1-8.

5. Stansell, P. Distributions of freak wave heights measured in the North Sea. Applied Ocean Research 2004, $26,35-48$.

6. Dysthe, K.; Krogstad, H.E.; Müller, P. Oceanic rogue waves. Annu. Rev. Fluid Mech. 2008, 40, $287-310$.

7. Weisse, R. Marine climate and climate change: storms, wind waves and storm surges; Springer Science \& Business Media, 2010.

8. Solli, D.; Ropers, C.; Koonath, P.; Jalali, B. Optical rogue waves. Nature 2007, 450, 1054.

9. Stenflo, L.; Marklund, M. Rogue waves in the atmosphere. Journal of Plasma Physics 2010, 76, $293-295$.

10. Moslem, W.; Shukla, P.; Eliasson, B. Surface plasma rogue waves. EPL (Europhysics Letters) 2011, 96, 25002.

11. Kibler, B.; Fatome, J.; Finot, C.; Millot, G.; Dias, F.; Genty, G.; Akhmediev, N.; Dudley, J.M. The Peregrine soliton in nonlinear fibre optics. Nature Physics 2010, 6, 790.

12. Levi-Civita, T. Determination rigoureuse des ondes permanentes d'ampleur finie. Mathematische Annalen 1925, 93, 264-314.

13. Nekrasov, A. On waves of permanent type. Izv. Ivanovo-Voznesensk. Politekhn. Inst 1921, 3, 52-65.

14. Smith, R. Giant waves. Journal of Fluid Mechanics 1976, 77, 417-431.

15. Zakharov, V.E. Collapse of Langmuir waves. Sov. Phys. JETP 1972, 35, 908-914.

16. Dai, C.Q.; Wang, Y.Y.; Tian, Q.; Zhang, J.F. The management and containment of self-similar rogue waves in the inhomogeneous nonlinear Schrödinger equation. Annals of Physics 2012, 327, 512-521.

17. Akhmediev, N.; Soto-Crespo, J.M.; Ankiewicz, A. Extreme waves that appear from nowhere: on the nature of rogue waves. Physics Letters A 2009, 373, 2137-2145.

18. Akhmediev, N.; Ankiewicz, A.; Soto-Crespo, J. Rogue waves and rational solutions of the nonlinear Schrödinger equation. Physical Review E 2009, 80, 026601.

19. Akhmediev, N.; Ankiewicz, A.; Soto-Crespo, J.; Dudley, J.M. Rogue wave early warning through spectral measurements? Physics Letters A 2011, 375, 541-544.

20. Chabchoub, A.; Hoffmann, N.; Branger, H.; Kharif, C.; Akhmediev, N. Experiments on wind-perturbed rogue wave hydrodynamics using the Peregrine breather model. Physics of Fluids 2013, 25, 101704.

21. Cousins, W.; Sapsis, T.P. Quantification and prediction of extreme events in a one-dimensional nonlinear dispersive wave model. Physica D: Nonlinear Phenomena 2014, 280, 48-58.

22. Cousins, W.; Sapsis, T.P. Unsteady evolution of localized unidirectional deep-water wave groups. Physical Review E 2015, 91, 063204.

23. Cousins, W.; Sapsis, T.P. Reduced-order precursors of rare events in unidirectional nonlinear water waves. Journal of Fluid Mechanics 2016, 790, 368-388. 
24. Dai, C.; Wang, Y.; Yan, C. Chirped and chirp-free self-similar cnoidal and solitary wave solutions of the cubic-quintic nonlinear Schrödinger equation with distributed coefficients. Optics Communications 2010, 283, 1489-1494.

25. Haghgoo, S.; Ponomarenko, S.A. Self-similar pulses in coherent linear amplifiers. Optics Express 2011, 19, 9750-9758.

26. Kruglov, V.; Peacock, A.; Harvey, J. Exact self-similar solutions of the generalized nonlinear Schrödinger equation with distributed coefficients. Physical Review Letters 2003, 90, 113902.

27. Kruglov, V.; Peacock, A.; Harvey, J. Exact solutions of the generalized nonlinear Schrödinger equation with distributed coefficients. Physical Review E 2005, 71, 056619.

28. Fermann, M.; Kruglov, V.; Thomsen, B.; Dudley, J.; Harvey, J. Self-similar propagation and amplification of parabolic pulses in optical fibers. Physical Review Letters 2000, 84, 6010.

29. Hamedi, H.R. Optical bistability and multistability via magnetic field intensities in a solid. Applied optics 2014, 53, 5391-5397.

30. Munk, W.; Snodgrass, F. Measurements of southern swell at Guadalupe Island. Deep Sea Research (1953) 1957, 4, 272-286.

31. Kruglov, V.; Peacock, A.; Dudley, J.; Harvey, J. Self-similar propagation of high-power parabolic pulses in optical fiber amplifiers. Optics letters 2000, 25, 1753-1755.

32. Osborne, A.R.; Onorato, M.; Serio, M. The nonlinear dynamics of rogue waves and holes in deep-water gravity wave trains. Physics Letters A 2000, 275, 386-393.

33. Zakharov, V.E. Stability of periodic waves of finite amplitude on the surface of a deep fluid. Journal of Applied Mechanics and Technical Physics 1968, 9, 190-194.

34. Serkin, V.; Hasegawa, A.; Belyaeva, T. Nonautonomous solitons in external potentials. Physical Review Letters 2007, 98, 074102 .

35. Dai, C.Q.; Wang, D.S.; Wang, L.L.; Zhang, J.F.; Liu, W. Quasi-two-dimensional Bose-Einstein condensates with spatially modulated cubic-quintic nonlinearities. Annals of Physics 2011, 326, 2356-2368.

36. Peregrine, D. Water waves, nonlinear Schrödinger equations and their solutions. The ANZIAM Journal 1983, 25, 16-43.

37. Zakharov, V.; Shabat, A. Interaction between solitons in a stable medium. Sov. Phys. JETP 1973, 37, $823-828$.

38. Matveev, V.B.; Matveev, V. Darboux transformations and solitons 1991.

39. Akhmediev, N.; Korneev, V. Modulation instability and periodic solutions of the nonlinear Schrödinger equation. Theoretical and Mathematical Physics 1986, 69, 1089-1093.

40. Dysthe, K.B.; Trulsen, K. Note on breather type solutions of the NLS as models for freak-waves. Physica Scripta 1999, 1999, 48.

41. Voronovich, V.V.; Shrira, V.I.; Thomas, G. Can bottom friction suppress 'freak wave'formation? Journal of Fluid Mechanics 2008, 604, 263-296.

42. Benjamin, T.B.; Feir, J. The disintegration of wave trains on deep water Part 1. Theory. Journal of Fluid Mechanics 1967, 27, 417-430.

43. Bespalov, V.; Talanov, V. Filamentary structure of light beams in nonlinear liquids. ZhETF Pisma Redaktsiiu 1966, 3, 471 .

44. Kim, D.S.; Markowsky, G.; Lee, S.G. Mobile Sage-Math for linear algebra and its application. Electronic Journal of Mathematics and Technology 2010, 4, 285-298.

45. Stein, W.; others. SageMath Mathematics Software (Version 6.5), 2015.

46. Kharif, C.; Pelinovsky, E. Physical mechanisms of the rogue wave phenomenon. European Journal of Mechanics-B/Fluids 2003, 22, 603-634.

47. Matsuno, Y. Bilinear transformation method; Elsevier, 1984.

48. Hirota, R. A new form of Bäcklund transformations and its relation to the inverse scattering problem. Progress of Theoretical Physics 1974, 52, 1498-1512.

49. Matveev, V.B. Positons: slowly decreasing analogues of solitons. Theoretical and Mathematical Physics 2002, 131, 483-497.

50. Osborne, A. Soliton physics and the periodic inverse scattering transform. Physica D: Nonlinear Phenomena $1995,86,81-89$.

51. Osborne, A. Solitons in the periodic Korteweg-de Vries equation, the FTHETA-function representation, and the analysis of nonlinear, stochastic wave trains. Physical Review E 1995, 52, 1105. 
52. Dysthe, K.B. Note on a modification to the nonlinear Schrödinger equation for application to deep water waves. Proc. R. Soc. Lond. A 1979, 369, 105-114.

53. Longuet-Higgins, M. The instability of gravity waves of infinite amplitude in deep water. II. Subharmonics 1978.

54. Trulsen, K.; Dysthe, K. Freak waves-a three-dimensional wave simulation. Proceedings of the 21st Symposium on naval Hydrodynamics. National Academy Press, 1997, Vol. 550, p. 558.

55. Trulsen, K.; Dysthe, K.B. A modified nonlinear Schrödinger equation for broader bandwidth gravity waves on deep water. Wave motion 1996, 24, 281-289.

56. Trulsen, K.; Kliakhandler, I.; Dysthe, K.B.; Velarde, M.G. On weakly nonlinear modulation of waves on deep water. Physics of fluids 2000, 12, 2432-2437.

57. Majda, A.; McLaughlin, D.; Tabak, E. A one-dimensional model for dispersive wave turbulence. Journal of Nonlinear Science 1997, 7, 9-44.

58. Pushkarev, A.; Zakharov, V. Quasibreathers in the MMT model. Physica D: Nonlinear Phenomena 2013, 248, 55-61.

59. Zakharov, V.; Dias, F.; Pushkarev, A. One-dimensional wave turbulence. Physics Reports 2004, 398, 1-65.

60. Zakharov, V.; Guyenne, P.; Pushkarev, A.; Dias, F. Wave turbulence in one-dimensional models. Physica D: Nonlinear Phenomena 2001, 152, 573-619.

61. Komen, G.J.; Cavaleri, L.; Donelan, M. Dynamics and modelling of ocean waves; Cambridge university press, 1996.

62. Lavrenov, I. The wave energy concentration at the Agulhas current off South Africa. Natural hazards 1998, 17, 117-127.

63. Zakharov, V.; Kuznetsov, E. Optical solitons and quasisolitons. Journal of Experimental and Theoretical Physics 1998, 86, 1035-1046.

64. Tao, Y.; He, J. Multisolitons, breathers, and rogue waves for the Hirota equation generated by the Darboux transformation. Physical Review E 2012, 85, 026601.

65. Ablowitz, M.J.; Kaup, D.J.; Newell, A.C.; Segur, H. Method for solving the sine-Gordon equation. Physical Review Letters 1973, 30, 1262.

66. Ankiewicz, A.; Soto-Crespo, J.; Akhmediev, N. Rogue waves and rational solutions of the Hirota equation. Physical Review E 2010, 81, 046602.

67. He, J.; Zhang, H.; Wang, L.; Porsezian, K.; Fokas, A. Generating mechanism for higher-order rogue waves. Physical Review E 2013, 87, 052914.

68. Ablowitz, M.J.; Musslimani, Z.H. Integrable nonlocal nonlinear Schrödinger equation. Physical review letters 2013, 110, 064105.

69. Ablowitz, M.J.; Musslimani, Z.H. Inverse scattering transform for the integrable nonlocal nonlinear Schrödinger equation. Nonlinearity 2016, $29,915$.

70. Ablowitz, M.J.; Musslimani, Z.H. Integrable nonlocal nonlinear equations. Studies in Applied Mathematics 2017, 139, 7-59.

71. Ablowitz, M.J.; Musslimani, Z.H. Integrable discrete P T symmetric model. Physical Review E 2014, 90, 032912.

72. Ablowitz, M.J.; Chakravarty, S.; Takhtajan, L.A. A self-dual Yang-Mills hierarchy and its reductions to integrable systems in 1+1 and 2+1 dimensions. Communications in Mathematical Physics 1993, 158, 289-314. doi:10.1007/BF02108076.

73. Ablowitz, M. MJ Ablowitz, DJ Kaup, AC Newell, and H. Segur, Stud. Appl. Math. 53, 249 (1974). Stud. Appl. Math. 1974, 53, 249.

74. Ablowitz, M.J.; Musslimani, Z.H. Integrable nonlocal nonlinear equations. Studies in Applied Mathematics 2017, 139, 7-59.

75. Yang, B.; Yang, J. General rogue waves in the nonlocal PT-symmetric nonlinear Schrodinger equation. arXiv preprint arXiv:1711.05930 2017.

76. Yu, F. Dynamics of nonautonomous discrete rogue wave solutions for an Ablowitz-Musslimani equation with PT-symmetric potential. Chaos: An Interdisciplinary Journal of Nonlinear Science 2017, 27, 023108. 